\title{
Elimination of background in film-based applications
}

\author{
Rachael M. Berry ${ }^{1}$, Stefan Schmitz², Brian D. Johnson ${ }^{1}$, Boguslawa Dworecki ${ }^{1}$, \\ Elizabeth A. Fritz ${ }^{3}$, Kenneth A. Roebuck ${ }^{4}$, and Kimberly K. Hines ${ }^{1}$
}

BioTechniques 37:298-302 (August 2004)

Erase-It ${ }^{\circledR}$ Background Eliminator is a solution used directly on processed film to remove background or improve data resolution. Traditional methods, such as optimization of the scientific protocol or better estimation of exposure time, are tedious and uncertain. Nevertheless, autoradiography continues to be a simple, effective method to visualize data. Therefore, we evaluated the ability of Erase-It Working Solution to help solve background and resolution issues. To demonstrate the efficiency of the Background Eliminator, we analyzed the product's ability to remove signal evenly, performance on several brands of film, and usefulness with various detection methods. Even reduction of signal was demonstrated by performing densitometric analysis on film generated from a dot blot with serial dilutions of analyte. In addition, overexposed films from various suppliers were effectively treated to remove background and visualize data. Autoradiographs, generated with ${ }^{32}$ P-labeled probes, and chemiluminescent substrate were also processed resulting in clearer images. Our results demonstrate that film data can be treated quickly and conveniently without fear of artificial enhancement. We show the Background Eliminator to be a universal and timesaving tool to visualize results that otherwise may be difficult to interpret.

\section{INTRODUCTION}

Numerous scientific techniques, including Southern blot analysis, electrophoretic mobility shift assays (EMSA), Northern blot analysis, ribonuclease protection assays, Western blot analysis, and in-gel detection utilize film as a lowcost imaging method. Background, however, is a persistent problem (1-4). To increase sensitivity and reduce background, several variables, including probe concentration, probe label, buffers, or substrate, can be optimized $(1,2,4,5)$. In addition, data resolution, or the ability to clearly distinguish data, can also be affected by various factors, such as the type and thickness of the film's emulsion, the distance between the sample and emulsion, or the exposure and the development time $(3,6-8)$. Along with the required experimental optimization, film quality and handling before and during development of the silver halide emulsion can contribute to background (3,68). Traditionally, many biochemical methods have been based upon detection with radiolabeled probes due to sensitive detection easily visualized by autoradiography $(3,7,9)$. Since radioactive emission occurs in all directions, overexposure can produce saturation of the film leading to a loss of resolution and difficulties in data interpretation (3,6-8). In addition, hours or days of exposure time are often required for radioisotopic detection, which makes acquiring the ideal image a time-consuming endeavor $(3,7)$. In recent years, enzyme-labeled probes and chemiluminescent substrates have become a safe practical alternative to traditional radiolabeled probes for detection in many systems and, when optimized, can produce results with high sensitivity and low background with generally shorter exposure time $(5,10-12)$. Depending upon the film, the linear dynamic range falls between 1.5 and 3 logs of activity, with the upper limit of detection being a completely black image (3). Sensitivity is not only limited by film quality, which is characterized by emulsion thickness and silver halide crystal size, but also by distance between the sample and film emulsion, the thickness of the sample source, and length of exposure (3,7). Additionally, the exposure time can be affected by the temperature, hydration or dehydration of gels, and quenching by protein stains (9). Chemical imperfections, pressure, exposure to heat and/or light, and even prolonged exposure to the safe-light are sources of background unrelated to the scientific method or mode of detection $(7,8)$.

Regardless of the cause of background or poor resolution, Erase-It ${ }^{\circledR}$ Background Eliminator (Pierce Biotechnology, Rockford, IL, USA) may be the ideal solution to salvage otherwise unreadable images. The Erase-It Working Solution reduces signal across the film, reducing background and/or speckling, and improving the resolution of overexposed bands. Incubations can be performed conveniently outside of the darkroom, in seconds to minutes, on wet or dry films freshly or previously developed. As an ideal solution, the Background Eliminator must remove signal evenly across the film, be capable of working on film from different manufacturers, and be useful for images generated by various detection methods. To determine whether the signal decrease is uniform, dot blots were prepared, incubated with Erase-It Working Solution, and scanned for densitometric analysis.

${ }^{1}$ Pierce Biotechnology, Rockford, IL, USA, ${ }^{2}$ Perbio Science, Bonn, Germany, ${ }^{3}$ Rush University, Chicago, IL, and ${ }^{4}$ National Institutes of Health, Bethesda, MD, USA 
To ensure the general utility of the product, films from separate suppliers were successfully treated with the Background Eliminator (data not shown). Autoradiographs generated with radiolabeled probes, as well as with enhanced chemiluminescence, were analyzed to demonstrate the flexibility of the Background Eliminator regardless of the assay or detection method. The results demonstrate that the Background Eliminator is a widely applicable solution for the treatment of film, saving valuable research time.

\section{MATERIALS AND METHODS}

\section{Preparation of Erase-It Working Solution and Treatment of Film}

For a $5 \times 7$-inch film, Erase-It Working Solution was prepared by combining $10 \mathrm{~mL}$ of Reagent A, $250 \mathrm{~mL}$ of purified water, and $10 \mathrm{~mL}$ of Reagent B. Volumes were adjusted in proportion to film size. Film was submerged in working solution and gently agitated to prevent sticking to the bottom of the container. The film was turned over every $15-30 \mathrm{~s}$ and monitored closely for the desired image. When achieved, the film was removed from the working solution and washed twice with water to stop the process. Close observation and thorough washing will avoid complete clearing of the film and the consequent loss of data.

\section{Densitometric Analysis}

Dot blots were prepared by spotting $1 \mu \mathrm{L}$ of 4 -fold serial dilutions ( $1 \mu \mathrm{g} / \mathrm{mL}$ to $15.63 \mathrm{ng} / \mathrm{mL})$ of biotinylated bovine serum albumin (B-BSA) (Pierce Biotechnology) onto nitrocellulose membrane (Pierce Biotechnology). The membrane was blocked for one hour using SuperBlock ${ }^{\circledR}$ Blocking Buffer in phosphate-buffered saline (PBS) (Pierce Biotechnology) plus $0.05 \%$ Tween ${ }^{\circledR}-20$ (SBPT). The membrane was probed with 1/50,000 streptavidin-horseradish peroxidase (SA-HRP; Pierce Biotechnology) in SBPT for $1 \mathrm{~h}$. Excess SA-HRP was removed by washing the blot six times in 5-min increments in PBS plus $0.05 \%$ Tween-20 (PBST). The blot was incubated in SuperSignal ${ }^{\circledR}$ West Pico Working Solution (Pierce Biotechnology) for 5 min before being exposed to CL-XPosure ${ }^{\mathrm{TM}}$ Film (Pierce Biotechnology) for $5 \mathrm{~min}$. The film was developed using GBX Developer and Replenisher and fixed using GBX Fixer and Replenisher (all from Eastman Kodak, Rochester, NY, USA). The film was cut into four strips, containing triplicates of each concentration. One strip was not treated with Erase-It Working Solution, one strip was treated for $1 \mathrm{~min}$, another strip was treated for $2.5 \mathrm{~min}$, and the last strip was treated for $4 \mathrm{~min}$. Each strip was scanned using Model GS-700 ${ }^{\mathrm{TM}}$ Imaging Densitometer (Bio-Rad Laboratories, Hercules, CA, USA).

\section{Applicability with Radioisotope or Chemiluminescent Detection}

Protocol for in-gel detection. Protein samples were electrophoresed using a 4\%-20\% Tris-glycine gel (Invitrogen, Carlsbad, CA, USA). The gel was removed from the plastic cassette, 
pretreated in $50 \%$ isopropyl alcohol for $15 \mathrm{~min}$, and rehydrated in purified water for $15 \mathrm{~min}$. At room temperature, the gel was incubated in biotinylated lysate probe at a 1:250 dilution for 1 $\mathrm{h}$ and washed three times for $10 \mathrm{~min}$ each with PBST. Next, the gel was incubated in a 1:10,000 dilution of SA-HRP for $1 \mathrm{~h}$ at room temperature and then washed three times for 10 min in PBST. The gel was incubated in UnBlot ${ }^{\circledR}$ Chemiluminescent Substrate (Pierce Biotechnology) working solution for $5 \mathrm{~min}$, followed by a 15-s water rinse, placed in a protective plastic sheet, and exposed to CL-XPosure Film. Initially, the film was treated for $2 \mathrm{~min}$ with fresh Erase-It Working Solution and scanned. The film was then treated at 1-min intervals with fresh Working Solution and scanned.

Electrophoretic mobility shift assay. An EMSA was performed on nuclear protein extracts from cells pretreated with inhibitors by the method of Osborn et al. and hybridized with a 100-fold molar excess of specific and nonspecific ${ }^{32} \mathrm{P}$-endlabeled oligonucleotide probes for 20-30 min at room temperature (13). Antibodies from Santa Cruz Biotechnology (Santa Cruz, CA, USA) were incubated in the binding reaction of two columns for super-shift assays. Protein-DNA and protein-DNA-antibody complexes were resolved on a $5 \%$ polyacrylamide gel. The gel was dried and exposed to Super RX film at $-70^{\circ} \mathrm{C}$, and the film was treated at 30 -s intervals with fresh Erase-It Working Solution and scanned.

\section{RESULTS AND DISCUSSION}

Film is commonly used to capture the results from many scientific methods including EMSA, UnBlot In-Gel Detection (Pierce Biotechnology), and Western, Northern, and Southern blot analyses. Following a laborious procedure, results may be developed only to discover background problems that may prevent interpretation of data. In radioactive and nonradioactive systems alike, background or poor resolution can arise from inadequate washing, inappropriate concentration of probes/antibodies, nonspecific binding of probes/ antibodies, or excessive exposure times (2-8). Previously, few options existed to rescue data from background, overexposure, or speckling. Although not always feasible, reexposing in hopes of attaining a more suitable image or repeating the entire experiment with minor optimizations have been the most common alternatives $(2,3)$. Another well-known method to remove background, the use of bleach, can be difficult to perform and control. We have demonstrated that Erase-It Background Eliminator is an effective tool to easily visualize captured data within minutes without the conjecture or repetition.

Ensuring that signal is removed uniformly is critical for the scientific use of the Background Eliminator. For example, if signal were removed at a higher rate from denser areas of the film, the signal-to-noise ratio would be reduced leading to a loss of resolution. Conversely, if signal were removed at a higher rate from less dense areas of the film, the signal-tonoise ratio would be artificially enhanced. To determine if the Background Eliminator reduces signal uniformly over time, B-BSA was spotted on a membrane at 1000, 250, 62.5, and

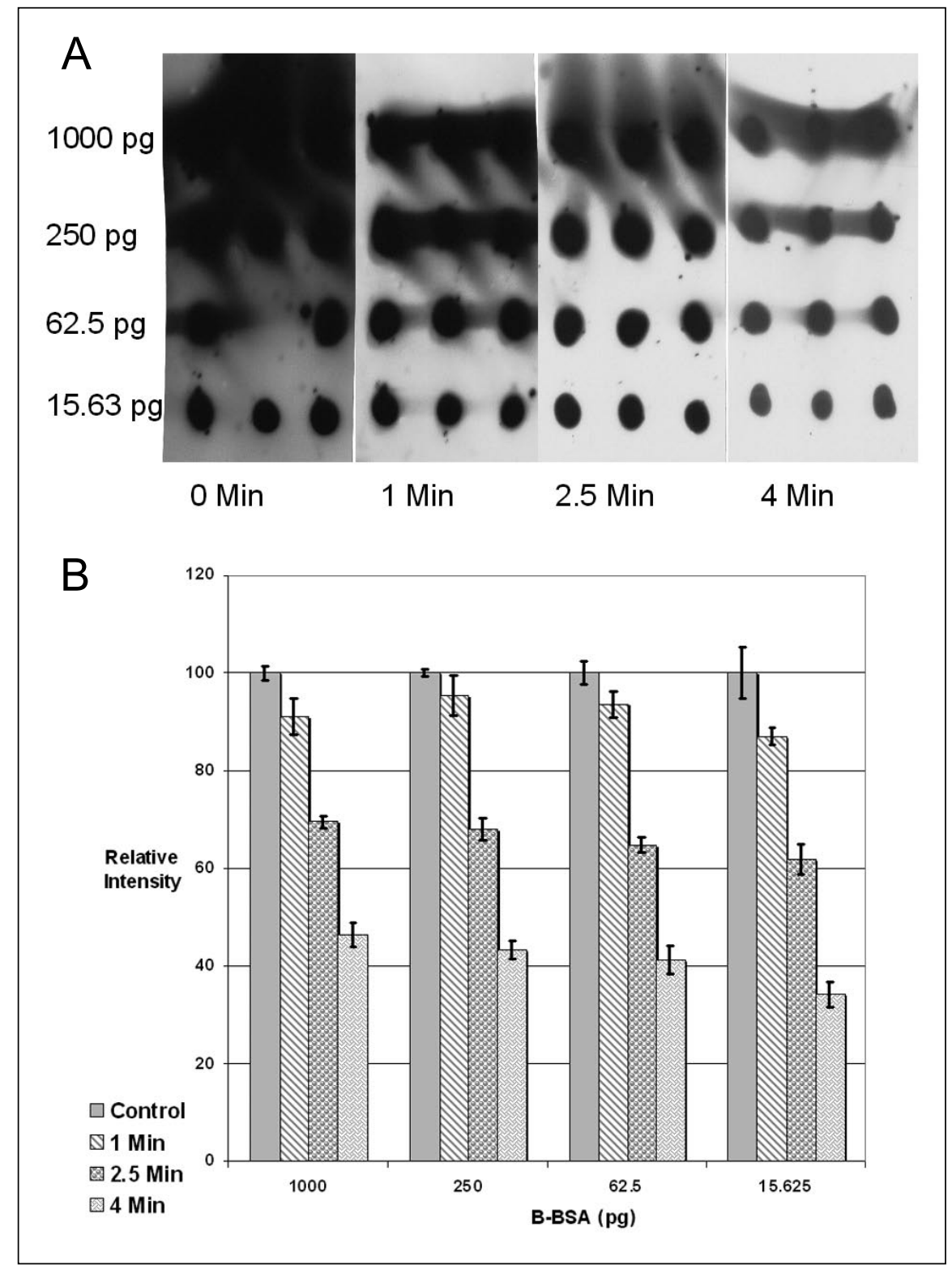

Figure 1. Signal intensity over time with use of Erase-It Background Eliminator. (A) Dot blots of biotinylated-bovine serum albumin (B-BSA) at 1000, 250, 62.5, and $15.63 \mathrm{pg}$ in triplicate untreated and treated with Erase-It Working Solution for 1, 2.5, or 4 min that were scanned for densitometric analysis. (B) Graph from densitometric analysis with Molecular Analyst ${ }^{\circledR}$ Software (Bio-Rad Laboratories) of relative intensity across the various concentrations shows a consistent decrease, indicating signal is removed evenly across film. 


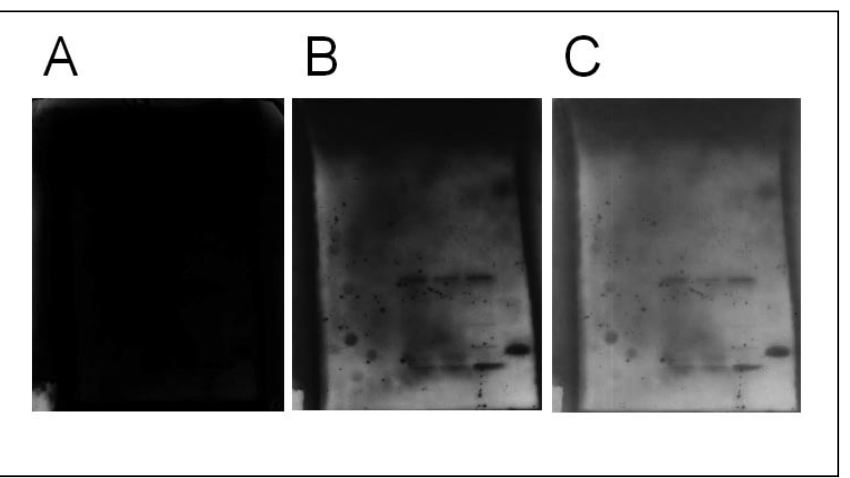

Figure 2. Effect of Erase-It Background Eliminator with chemiluminescent substrate. (A) Using chemiluminescent detection, the film produced using UnBlot In-Gel Detection showed no discernable bands. (B) However, following $4 \mathrm{~min}$ of treatment, background was reduced allowing bands to be identified. (C) To ascertain the presence of bands in the upper portion of the film, incubation was continued for a total of $5 \mathrm{~min}$ to further reduce background.

$15.63 \mathrm{pg}$, followed by incubation with SA-HRP and SuperSignal West Pico Substrate for detection. Film was exposed to the dot blot for $5 \mathrm{~min}$, developed, and divided into sections prior to treatment with Erase-It Working Solution. The film sections were treated for $0,1,2.5$, or 4 min with the Work- ing Solution and then scanned for densitometric analysis. Visually, the reduction of background was easily noted at each time point, and the resolution of individual spots was improved following incubation (Figure 1A). Densitometric analysis was used to calculate the relative intensity of each spot to show the reduction of signal over time (Figure 1B). The results demonstrate that at each concentration the relative intensity or reduction of signal was similar. For example, relative intensity decreased from $100 \%$ to $69.5 \%$ at $1000 \mathrm{pg}$, $68.0 \%$ at $250 \mathrm{pg}, 64.8 \%$ at $62.5 \mathrm{pg}$, and $61.9 \%$ at $15.63 \mathrm{pg}$ after 2.5 min of treatment with the Working Solution. Because film has a limited dynamic range, the observed decrease in relative intensity appears lower at higher B-BSA concentrations; however, this result is skewed due to saturation of the film at higher concentrations. Although signal is removed evenly from film, an overexposed film, even after treatment with the Background Eliminator, may provide data that cannot be accurately quantitated using densitometric analysis. Also, note that low-resolution bands or the entire image can be completely removed, so constant supervision is required during the treatment, and scanning the film after sequential incubations is recommended. Overall, the parallel decrease over time in the relative intensity of data points at various concentrations demonstrates uniform reduction of signal across the film. 


\section{Product Application Focus}

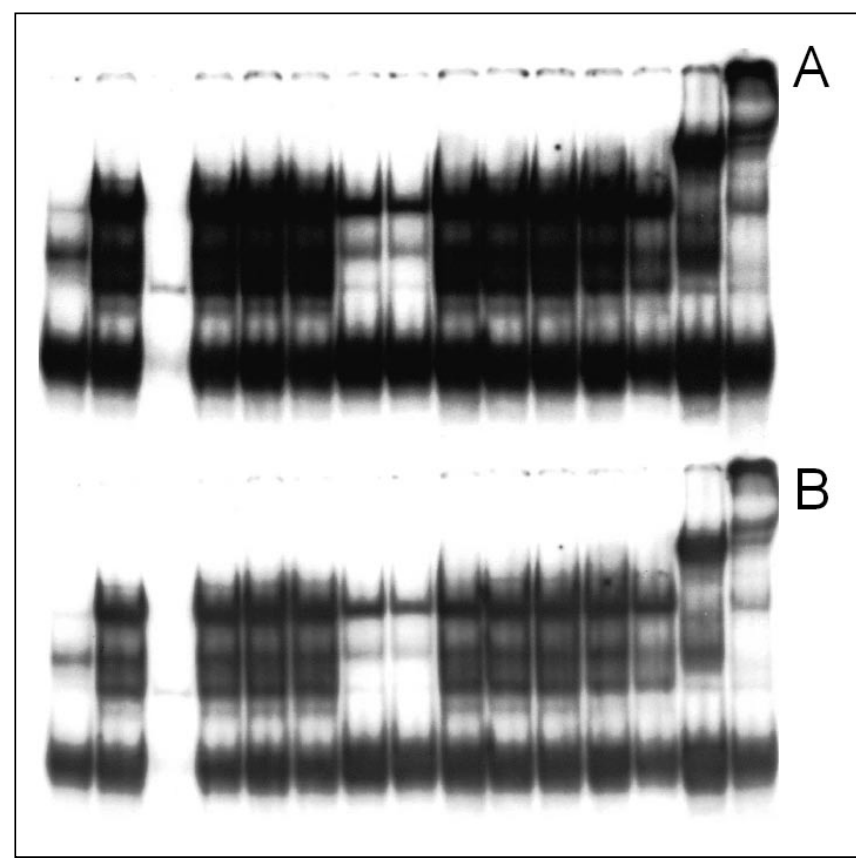

Figure 3. Improved resolution of radioisotopic data using Erase-It Background Eliminator. (A) Electrophoretic mobility shift assay (EMSA) of nuclear protein extract incubated with inhibitors and detected using ${ }^{32} \mathrm{P}$-end-lableled oligonucleotide probes. (B) In 2 min with use of Erase-It Background Eliminator, overblown bands were minimized to improve resolution.

In order to illustrate compatibility with common scientific methods, Erase-It Background Eliminator was used on both film generated using UnBlot In-Gel Detection of proteins with a chemiluminescent substrate and film generated from a gel shift assay with ${ }^{32} \mathrm{P}$-end-labeled oligonucleotide probes. Film from the initial exposure generated with UnBlot In-Gel Detection displayed no discernable data (Figure 2A). The film was placed in Erase-It Working Solution for various incubation times. Following 4 min of treatment, the background was reduced, allowing interpretation of the data (Figure 2B). Incubation was continued for an additional $1 \mathrm{~min}$, for a total of $5 \mathrm{~min}$, to reduce the background in the upper portion of the film and ensure that no further data was still obscured (Figure 2C). Similarly, results were generated using EMSA and ${ }^{32} \mathrm{P}$-end-labeled oligonucleotide probes for detection. Initial results were ambiguous due to overexposure and poor band resolution (Figure $3 \mathrm{~A})$. However, using Erase-It Working Solution for $2 \mathrm{~min}$ reduced the background and improved the resolution (Figure 3B) allowing visualization of specific bands. These results indicate that the Background Eliminator is useful for improving chemiluminescent or radioisotopic images in minutes.

Autoradiographs, regardless of method generated, can be conveniently treated with the Background Eliminator to reveal data that may otherwise only be obtained by time-consuming reexposure or assay repetition. The Working Solution can also be used repeatedly on the same film until the optimal image is obtained. However, attentiveness and/or repeat scans are critical to avoid the loss of less intense data. In addition, densitometric analysis demonstrated that signal was removed evenly across the film. Use does not require the purchase of special film (data not shown), the alteration of current sci- entific protocols, or a darkroom. Notably, film can be pulled from laboratory notebooks and treated years after experiment completion (data not shown). In conclusion, Erase-It Background Eliminator provides an innovative option to researchers and the flexibility to rescue results within minutes.

\section{ACKNOWLEDGMENTS}

We are extremely grateful to Drs. Mahesh Mathrubutham and Yvonne B. Sullivan for their helpful suggestions with manuscript preparation.

\section{COMPETING INTERESTS STATEMENT}

E.A.F. and K.A.R. declare no competing interests, and B.D.J., B.D., and K.K.H. declare that they are currently employed by Pierce Biotechnology, the manufacturer and distributer of Erase-It ${ }^{\circledR}$ Background Eliminator.

\section{REFERENCES}

1.Kelsell, R.E. 1996.Hybridization and competition hybridization of Southern blots, p.31-39. In A.J. Harwood (Ed.), Methods in Molecular Biology, Vol. 58: Basic DNA and RNA Protocols. Humana Press, Totowa.

2.Pampori, N.A., M.K. Pampori, and B.H. Shapiro. 1995. Dilution of the chemiluminescence reagents reduces the background noise on western blots. BioTechniques 18:588-590.

3.Upham, L.V. and D.F. Englert. 1998. Radionuclide imaging, p. 647-692. In M.F. L'Annunziata (Ed.), Handbook of Radioactivity Analysis. Academic Press, San Diego.

4.Wu, M., P.G. Stockley, and W.H. Martin II. 2002. An improved Western blotting technique effectively reduces background. Electrophoresis 23:2373-2376.

5.Ji, X., K. Lee, and B. DiPaolo. 2002. High sensitivity hybridization assay for quantitation of residual $E$. coli DNA. BioTechniques 32:1162-1167.

6.L'Annunziata, M.F. 1987. Radionuclide Tracers: Their Detection and Measurement. Academic Press, Orlando.

7.Rogers, A.W. 1969. Techniques of Autoradiography. Elsevier Publishing Company, Amsterdam.

8.Pelc, S.R. 1972. Theory of Autoradiography, p.1-17. In P.B. Gahan (Ed.), Autoradiography for Biologists. Academic Press, New York.

9.Merril, C. and K. Washart. 1998. Protein detection methods, p.53-91. In B.D. Hames (Ed), Gel Electrophoresis of Proteins: A Practical Approach Oxford University Press, New York.

10.Accotto, G.P., A.M. Vaira, E. Noris, and M. Vecchiati. 1998. Using non-radioactive probes on plants; a few examples. J. Biolumin. Chemilumin. 13:295-301.

11.Bakkali, L., R. Guillou, M. Gonzague, and C. Cruciere. 1994. A rapid and sensitive chemiluminescence dot-immunobinding assay for screening hybridoma supernatants. J. Immunol. Methods 170:177-184.

12.Engler-Blum, G., M. Meier, J. Frank, and G.A. Müller. 1993. Reduction of background problems in nonradioactive northern and Southern blot analyses enables higher sensitivity than ${ }^{32} \mathrm{P}$-based hybridizations. Anal. Biochem. 210:235-244.

13. Osborn, L., S. Kunkel, and G.J. Nabel. 1989. Tumor necrosis factor alpha and interleukin 1 stimulate the human immunodeficiency virus enhancer by activation of the nuclear factor kappa B. Proc. Natl. Acad. Sci. USA 86:2336-2340.

Address correspondence to Rachael M. Berry, Research and Development, Pierce Biotechnology, Inc., $3747 \mathrm{~N}$. Meridian Rd., Rockford, IL 61101, USA. e-mail: rachael. berry@piercenet.com 